\title{
Fuzzy fractional differential equations with Nagumo and Krasnoselskii-Krein condition
}

\author{
T. Allahviranloo ${ }^{1}$ S. Abbasbandy ${ }^{1}$ S. Salahshour ${ }^{2}$ \\ ${ }^{1}$ Department of Mathematics, Science and Research Branch, Islamic Azad University, Tehran, Iran. \\ ${ }^{2}$ Department of Mathematics, Mobarakeh Branch, Islamic Azad University, Mobarakeh, Iran.
}

\begin{abstract}
In this paper, we consider two new uniqueness results for fuzzy fractional differential equations (FFDEs) involving Riemann-Liouville generalized H-differentiability with the Nagumo-type condition and the Krasnoselskii-Krein-type condition. To this purpose, the equivalent integral forms of FFDEs are determined and then these are used to study the convergence of the Picard successive approximations.
\end{abstract}

Keywords: Fuzzy fractional differential equations, Riemann-Liouville generalized H-differentiability, Nagumo condition, Krasnoselskii-Krein condition.

\section{Introduction}

Fractional differential and integral equations play increasingly important roles in the modeling of engineering and science problems, as shown in $[1,6,7,9,17]$. It has been established that, in many situations, these models provide more suitable results than analogous models with integer derivatives. The calculus of fractional order derivatives and the theory of fractional differential equations has been studied comprehensively in $[2,3,4,13,14,15]$.

In order to investigate the solutions of fractional differential equations involving Riemann-Liouville differentiability in he real cases, we used the fuzzy concept in the structure of problem which leads to conversion of the original to the fuzzy fractional differential equations.

Consider the following fuzzy fractional differential equation of order $0<q<1$ with the initial condition

$$
\left\{\begin{array}{ccc}
\left({ }^{R L} D^{q} x\right)(t) & = & f(t, x) \\
x\left(t_{0}\right) & = & x^{0} \in \mathbb{E}
\end{array}\right.
$$

where $f:(a, b) \times \mathbb{E} \longrightarrow \mathbb{E}$ is a continuous fuzzyvalued function and $x^{0}=\left.x(t)\left(t-t_{0}\right)^{1-q}\right|_{t=t_{0}}$ with

$$
\begin{aligned}
\mathbb{E}_{0} & =\left\{(t, x): t_{0} \leq t \leq t_{0}+a, d\left(x(t), x^{0}(t)\right) \leq b\right\}, \\
x^{0}(t) & =\frac{x^{0}\left(t-t_{0}\right)^{q-1}}{\Gamma(q)} .
\end{aligned}
$$

where $d$ is Hausdorff distance. In this paper, we try to obtain two useful results about existence and uniqueness of solutions of (1) under a new definition for fuzzy fractional derivative about fractional order $0<q<1$, so-called Riemann-Liouville generalized H-differentiability. Notice that, with best of our knowledge this is first time that fuzzy differential equations are considered about fractional order.

The paper is organized as follows: in Section 2 , some basic concepts are given which will be used later. In Section 3, the Riemann-Liouville Hdifferentiability is proposed for fuzzy-valued functions and then some of important results of it are provided. In Section 4 and Section 5, the Nagumo condition and the Krasnoselskii-Krein condition are considered for studying the uniqueness property, respectively. Moreover, some results are derived in Section 6. The paper ends with conclusion.

\section{Basic concepts}

The basic definition of fuzzy numbers is given in [21].

We denote the set of all real numbers by $\mathbb{R}$ and the set of all fuzzy numbers on $\mathbb{R}$ is indicated by $\mathbb{E}$. A fuzzy number is a mapping $u: \mathbb{R} \rightarrow[0,1]$ with the following properties:
(a) $u$ is upper semi-continuous,
(b) $u$ is fuzzy convex, i.e., $u(\lambda x+(1-\lambda) y) \geq$ $\min \{u(x), u(y)\}$ for all $x, y \in \mathbb{R}, \lambda \in[0,1]$,
(c) $u$ is normal, i.e., $\exists x_{0} \in \mathbb{R}$ for which $u\left(x_{0}\right)=1$,
(d) supp $u=\{x \in \mathbb{R} \mid u(x)>0\}$ is the support of the $u$, and its closure $\mathrm{cl}(\operatorname{supp} \mathrm{u})$ is compact.
An equivalent parametric definition is also given in $[8,16]$ as follows:

Definition 1 A fuzzy number $u$ in parametric form is a pair $(\underline{u}, \bar{u})$ of functions $\underline{u}(r), \bar{u}(r), 0 \leq r \leq 1$, which satisfy the following requirements:

1. $\underline{u}(r)$ is a bounded non-decreasing left continuous function in $(0,1]$, and right continuous at 0 ,

2. $\bar{u}(r)$ is a bounded non-increasing left continuous function in $(0,1]$, and right continuous at 0 ,

3. $\underline{u}(r) \leq \bar{u}(r), 0 \leq r \leq 1$.

According to Zadeh's extension principle, the operation of addition on $\mathbb{E}$ is defined by

$(u+v)(x)=\sup _{y \in \mathbb{R}} \min \{u(y), v(x-y)\}, \quad x \in \mathbb{R}$, 
and scalar multiplication of a fuzzy number is given by

$$
(k \cdot u)(x)=\left\{\begin{array}{l}
u(x / k), \quad k>0, \\
\widetilde{0}, \quad k=0,
\end{array}\right.
$$

where $\tilde{0} \in \mathbb{E}$.

The Hausdorff distance between fuzzy numbers given by $d: \mathbb{E} \times \mathbb{E} \longrightarrow[0,+\infty]$,

$$
d(u, v)=\sup _{r \in[0,1]} \max \{|\underline{u}(r)-\underline{v}(r)|,|\bar{u}(r)-\bar{v}(r)|\},
$$

where $u=(\underline{u}(r), \bar{u}(r)), v=(\underline{v}(r), \bar{v}(r)) \subset \mathbb{R}$ is utilized in [5]. Then, it is easy to see that $d$ is a metric in $\mathbb{E}$ and has the following properties (see [18]):

(1) $\quad d(u+w, v+w)=d(u, v), \quad \forall u, v, w \in \mathbb{E}$,

(2) $\quad d(k u, k v)=|k| d(u, v), \quad \forall k \in \mathbb{R}, u, v \in \mathbb{E}$,

(2) $\quad d(u+v, w+e) \leq d(u, w)+d(v, e), \quad \forall u, v, w, e \in$ $\mathbb{E}$

(4) $(d, \mathbb{E})$ is a complete metric space.

Theorem 1 [19]. Let $f(x)$ be a fuzzy-valued function on $[a, \infty)$ represented by $(f(x ; r), \bar{f}(x ; r))$. For any fixed $r \in[0,1]$, assume $f(x ; r)$ and $\bar{f}(x ; r)$ are Riemann-integrable on $[a, b]$ for every $b \geq a$, and assume there are two positive functions $\underline{M}(r)$ and $\bar{M}(r)$ such that $\int_{a}^{b}|\underline{f}(x ; r)| d x \leq \underline{M}(r)$ and $\int_{a}^{b}|\bar{f}(x ; r)| d x \leq \bar{M}(r)$ for every $b \geq a$. Then $f(x)$ is improper fuzzy Riemann-integrable on $[a, \infty)$ and the improper fuzzy Riemann-integral is a fuzzy number. Further more, we have:

$$
\int_{a}^{\infty} f(x) d x=\left(\int_{a}^{\infty} \underline{f}(x ; r) d x, \int_{a}^{\infty} \bar{f}(x ; r) d x\right) .
$$

Definition 2 Let $x, y \in \mathbb{E}$. If there exists $z \in \mathbb{E}$ such that $x=y+z$, then $z$ is called the H-difference of $x$ and $y$, and it is denoted by $x \ominus y$.

In this paper, the sign " $\ominus$ " always stands for $\mathrm{H}$ difference, and also note that $x \ominus y \neq x+(-1) y$.

\section{Riemann-Liouville H-differentiability}

Now, we introduce the definition of fuzzy RiemannLiouville integrals and derivatives under Hukuhara difference. We try to produce such definitions and statements similar to the non-fractional one in fuzzy context [5].

Also, we denote by $C^{\mathbb{F}}[a, b]$ the space of all fuzzy-valued functions which are continuous and lebesgue measure on $[a, b]$ and we assumed that all fuzzy-valued functions in this paper are placed in $C^{\mathbb{F}}[a, b]$. Also, the fuzzy-valued functions in the paper are assumed to be definable in each space.

Now, we define the fuzzy Riemann-Liouville integral of fuzzy-valued function as follows:
Definition 3 Let $f:[a, b] \longrightarrow \mathbb{E}$. The fuzzy Riemann-Liouville integral of fuzzy-valued function $f$ is defined as follows:

$\left(I^{q} f\right)(x)=\frac{1}{\Gamma(q)} \int_{a}^{x} \frac{f(t) d t}{(x-t)^{1-q}}, \quad x>a, 0<q<1$.

Notice that, since $f$ is assumed to be integrable and $(x-t)^{q-1}$ is a crisp function, we deduce that $\frac{f(t)}{(x-t)^{1-q}}$ is integrable and then, the existence of integral (2) is proved.

Let us consider the r-cut representation of fuzzyvalued function $f$ is $f(x ; r)=[f(x ; r), \bar{f}(x ; r)]$, for $0 \leq r \leq 1$, then we can indicate the fuzzy RiemannLiouville integral of fuzzy-valued function $f$ based on its lower and upper functions as follows:

Theorem 2 Let $f:[a, b] \longrightarrow \mathbb{E}$. The fuzzy Riemann-Liouville integral of a fuzzy-valued function $f$ can be expressed as follows:

$\left(I^{q} f\right)(x ; r)=\left[\left(I^{q} \underline{f}\right)(x ; r),\left(I^{q} \bar{f}\right)(x ; r)\right], \quad 0 \leq r \leq 1$,

where

$$
\begin{aligned}
\left(I^{q} \underline{f}\right)(x ; r) & =\frac{1}{\Gamma(q)} \int_{a}^{x} \frac{f(t ; r) d t}{(x-t)^{1-q}} \\
\left(I^{q} \bar{f}\right)(x ; r) & =\frac{1}{\Gamma(q)} \int_{a}^{x} \frac{\bar{f}(t ; r) d t}{(x-t)^{1-q}}
\end{aligned}
$$

Now, we define fuzzy Riemann-Liouville fractional derivatives about order $0<q<1$ for fuzzyvalued function $f$ which is a direct extension of strongly generalized H-differentiability [5] in the fractional literature.

Definition 4 Let $f:(a, b) \longrightarrow \mathbb{E}, x_{0}$ in $(a, b)$ and $\Phi(x)=\frac{1}{\Gamma(1-q)} \int_{a}^{x} \frac{f(t) d t}{(x-t)^{q}}$. We say that $f(x)$ is Riemann-Liouville H-differentiable about order $0<q<1$ at $x_{0}$, if there exists an element $\left({ }^{R L} D_{a^{+}}^{q} f\right)\left(x_{0}\right) \in C^{\mathbb{F}}, 0<q<1$, such that for all $0 \leq r \leq 1, h>0$

$$
\begin{aligned}
\left({ }^{R L} D_{a^{+}}^{q} f\right)\left(x_{0}\right) & =\lim _{h \longrightarrow 0} \frac{\Phi\left(x_{0}+h\right) \ominus \Phi\left(x_{0}\right)}{h} \\
& =\lim _{h \longrightarrow 0} \frac{\Phi\left(x_{0}\right) \ominus \Phi\left(x_{0}-h\right)}{h},
\end{aligned}
$$

or

$$
\begin{aligned}
\left({ }^{R L} D_{a^{+}}^{q} f\right)\left(x_{0}\right) & =\lim _{h \longrightarrow 0} \frac{\Phi\left(x_{0}\right) \ominus \Phi\left(x_{0}+h\right)}{-h} \\
& =\lim _{h \rightarrow 0} \frac{\Phi\left(x_{0}-h\right) \ominus \Phi\left(x_{0}\right)}{-h} .
\end{aligned}
$$

For sake of simplicity, we say that a fuzzy-valued function $f$ is $(1, q)$-differentiable if it is differentiable as in the Definition 4 case $(1)$, and is $(2, q)$ differentiable if it is differentiable as in the Definition 4 case (2). 
Theorem 3 Let $f:(a, b) \longrightarrow \mathbb{E}, x_{0}$ in $(a, b)$ and $0<q<1$ for all $0 \leq r \leq 1$. Then:

(1) If $f(x)$ be a $(1, q)$-differentiable fuzzy-valued function, then:

$\left({ }^{R L} D_{a^{+}}^{q} f\right)\left(x_{0} ; r\right)=\left[\left({ }^{R L} D_{a^{+}}^{q} \underline{f}\right)\left(x_{0}, r\right),\left({ }^{R L} D_{a^{+}}^{q} \bar{f}\right)\left(x_{0}, r\right)\right]$.

(2) If $f(x)$ be a (2,q)-differentiable fuzzy-valued function, then:

$\left({ }^{R L} D_{a^{+}}^{q} f\right)\left(x_{0} ; r\right)=\left[\left({ }^{R L} D_{a^{+}}^{q} \bar{f}\right)\left(x_{0} ; r\right),\left({ }^{R L} D_{a^{+}}^{q} \underline{f}\right)\left(x_{0}, r\right)\right]$,

where

$\left({ }^{R L} D_{a^{+}}^{q} \underline{f}\right)\left(x_{0} ; r\right)=\left[\frac{1}{\Gamma(1-q)} \frac{d}{d x} \int_{a}^{x} \frac{f(t ; r) d t}{(x-t)^{q}}\right]_{x=x_{0}}$

and

$\left({ }^{R L} D_{a^{+}}^{q} \bar{f}\right)\left(x_{0} ; r\right)=\left[\frac{1}{\Gamma(1-q)} \frac{d}{d x} \int_{a}^{x} \frac{\bar{f}(t, r) d t}{(x-t)^{1-q}}\right]_{x=x_{0}}$

\section{Nagumo-type uniqueness for FFDEs}

In this section, we consider the Nagumo-type condition in order to obtain a new uniqueness result for FFDEs.

We shall first extend a Nagumo-type uniqueness result for (1).

Theorem 4 Assume that the fuzzy-valued function $f$ in (1) satisfies the following Nagumo-type condition:

$$
d(f(t, x), f(t, y)) \leq \frac{q \Gamma(q) d(x, y)}{\left(t-t_{0}\right)^{q}},
$$

for $t \neq t_{0},(t, x),(t, y) \in \mathbb{E}_{0}$. Then, the following successive approximations given by

$x_{n+1}(t)=x^{0}(t)+\frac{1}{\Gamma(q)} \int_{t_{0}}^{t}(t-s)^{q-1} f\left(s, x_{n}(s)\right) d s$

in the sense of $(1, q)$-differentiability, and

$\widehat{x}_{n+1}(t)=x^{0}(t) \ominus \frac{-1}{\Gamma(q)} \int_{t_{0}}^{t}(t-s)^{q-1} f\left(s, \widehat{x}_{n}(s)\right) d s$

in the sense of $(2, q)$-differentiability, converge to two unique solutions $x(t)$ and $\widehat{x}(t)$ of (1), respectively, on $\left[t_{0}, t_{0}+\eta\right]$ where $\eta=\min =$ $\left(a,\left(\frac{b \Gamma(1+q)}{M}\right)^{\frac{1}{q}}\right), M$ being the bound of $f$ on $\mathbb{E}_{0}$, i.e., $d(f, \widetilde{0}) \leq M$.

Proof. Let us consider $x(t), y(t)$ be any two $(1, q)$ solutions of (1) and $\widehat{x}(t), \widehat{y}(t)$ be any two $(2, q)$ solutions of (1). Set

$$
\begin{aligned}
\phi(t) & =d(x(t), y(t)), \\
\widehat{\phi}(t) & =d(\widehat{x}(t), \widehat{y}(t)) .
\end{aligned}
$$

Note that $\phi\left(t_{0}\right)=\widehat{\phi}\left(t_{0}\right)=0$. Also, we pointed out that:

$$
\begin{aligned}
& \lim _{t \rightarrow t_{0}^{+}} \frac{\phi(t)}{\left(t-t_{0}\right)^{q}}= \\
& \lim _{t \rightarrow t_{0}^{+}}\left(\frac{1}{\left(t-t_{0}\right) \Gamma(q)} \int_{t_{0}}^{t} \frac{d(f(s, x(s), f(s, y(s))) d s}{(t-s)^{-q+1}}\right)= \\
& \frac{1}{\Gamma(q)}\left\{\left(t-t_{0}\right)^{q-1} \cdot d\left(f\left(t_{0}, x\left(t_{0}\right), f\left(t_{0}, y\left(t_{0}\right)\right)\right)\right\}=0 .\right.
\end{aligned}
$$

and

$$
\begin{aligned}
& \lim _{t \rightarrow t_{0}^{+}} \frac{\widehat{\phi}(t)}{\left(t-t_{0}\right)^{q}}= \\
& \lim _{t \rightarrow t_{0}^{+}}\left(\frac{1}{\left(t-t_{0}\right) \Gamma(q)} \int_{t_{0}}^{t} \frac{d(f(s, \widehat{x}(s), f(s, \widehat{y}(s))) d s}{(t-s)^{-q+1}}\right)= \\
& \frac{1}{\Gamma(q)}\left\{\left(t-t_{0}\right)^{q-1} \cdot d\left(f\left(t_{0}, \widehat{x}\left(t_{0}\right), f\left(t_{0}, \widehat{y}\left(t_{0}\right)\right)\right)\right\}=0 .\right.
\end{aligned}
$$

Also,

$$
\begin{aligned}
& \lim _{t \rightarrow t_{0}^{+}} \frac{\phi(t)}{\left(t-t_{0}\right)^{q}}=\lim _{t \rightarrow t_{0}^{+}} \frac{\phi(t)}{\left(t-t_{0}\right)} \cdot\left(t-t_{0}\right)^{1-q}, \\
& \lim _{t \rightarrow t_{0}^{+}} \frac{\widehat{\phi}(t)}{\left(t-t_{0}\right)^{q}}=\lim _{t \rightarrow t_{0}^{+}} \frac{\widehat{\phi}(t)}{\left(t-t_{0}\right)} \cdot\left(t-t_{0}\right)^{1-q} .
\end{aligned}
$$

By using the fuzzy Nagumo-type condition (3), we get:

$$
\begin{aligned}
& \phi(t) \leq \frac{1}{\Gamma(q)} \int_{t_{0}}^{t} \frac{(t-s)^{q-1} \phi(s) q \Gamma(q) d s}{\left(s-t_{0}\right)^{q}}, \\
& \widehat{\phi}(t) \leq \frac{1}{\Gamma(q)} \int_{t_{0}}^{t} \frac{(t-s)^{q-1} \widehat{\phi}(s) q \Gamma(q) d s}{\left(s-t_{0}\right)^{q}} .
\end{aligned}
$$

Setting $\frac{\phi(t)}{\left(t-t_{0}\right)^{q}}=\psi(t)$ and $\frac{\widehat{\phi}(t)}{\left(t-t_{0}\right)^{q}}=\widehat{\psi}(t)$ and noting that $\psi\left(t_{0}\right)=\widehat{\psi}\left(t_{0}\right)=0$, Eqs.(8)-(9) reduce to the following relations, respectively:

$$
\begin{aligned}
& \psi(t) \leq \frac{q}{\left(t-t_{0}\right)^{q}} \cdot \int_{t_{0}}^{t}\left(t-t_{0}\right)^{q-1} \psi(s) d s, \\
& \widehat{\psi}(t) \leq \frac{q}{\left(t-t_{0}\right)^{q}} \cdot \int_{t_{0}}^{t}\left(t-t_{0}\right)^{q-1} \widehat{\psi}(s) d s .
\end{aligned}
$$

In order to establish uniqueness of solution $((1, q)$ or $(2, q)$-solution), we must show that $\psi(t)=\widehat{\psi}(t) \equiv$ 0 . If it is not true, let us consider

$$
\begin{aligned}
m & =\max _{\left[t_{0}, t_{0}+\eta\right]} \psi(t) \\
& =\psi\left(t_{1}\right), t_{1} \in\left[t_{0}, t_{0}+\eta\right], \\
\widehat{m} & =\max _{\left[t_{0}, t_{0}+\eta\right]} \widehat{\psi}(t) \\
& =\widehat{\psi}\left(t_{2}\right), t_{2} \in\left[t_{0}, t_{0}+\eta\right] .
\end{aligned}
$$


which lead to:

$$
\begin{aligned}
m=\psi\left(t_{1}\right) & \leq \frac{q}{\left(t_{1}-t_{0}\right)^{q}} \int_{t_{0}}^{t}\left(t_{1}-s\right)^{q-1} \psi(s) d s \\
& <\left.\frac{q m}{\left(t_{1}-t_{0}\right)^{q}} \frac{\left(t_{1}-s\right)^{q}}{-q}\right|_{t_{0}} ^{t_{1}} \\
& =\frac{q m}{\left(t_{1}-t_{0}\right)^{q}} \frac{\left(t_{1}-t_{0}\right)^{q}}{q}=m . \\
\widehat{m}=\widehat{\psi}\left(t_{1}\right) & \leq \frac{q}{\left(t_{1}-t_{0}\right)^{q}} \int_{t_{0}}^{t}\left(t_{1}-s\right)^{q-1} \widehat{\psi}(s) d s \\
& <\left.\frac{q \widehat{m}}{\left(t_{1}-t_{0}\right)^{q}} \frac{\left(t_{1}-s\right)^{q}}{-q}\right|_{t_{0}} ^{t_{1}} \\
& =\frac{q \widehat{m}}{\left(t_{1}-t_{0}\right)^{q}} \frac{\left(t_{1}-t_{0}\right)^{q}}{q}=\widehat{m} .
\end{aligned}
$$

These are contradiction and hence we get $\psi(t)=\widehat{\psi}(t) \equiv 0$. Therefore, obtained results imply that $\phi(t)=\widehat{\phi}(t) \equiv 0$, which lead to prove the uniqueness of $(1, q)$ - and $(2, q)$-solution.

Now, we investigate the following properties for both successive approximations $\left\{x_{n+1}\right\}$ and $\left\{\widehat{x}_{n+1}\right\}, n=0,1,2, \ldots$ :

- well defined and continuous,

- uniformly bounded and

- equicontinuous on $\left[t_{0}, t_{0}+\eta\right], \quad \eta=$ $\min \left(a,\left(\frac{b \Gamma(1+q)}{M}\right)^{\frac{1}{q}}\right), \quad d(f(t, x), \widetilde{0}) \leq M \quad$ on $\mathbb{E}_{0}$.

In view of (4) and (5), we have:

$$
\begin{aligned}
& d\left(x_{n+1}(t), x^{0}(t)\right) \leq \frac{1}{\Gamma(q)} \int_{t_{0}}^{t} \frac{d\left(f\left(s, x_{n}(x)\right), \widetilde{0}\right) d s}{(t-s)^{-q+1}}, \\
& d\left(\widehat{x}_{n+1}(t), x^{0}(t)\right) \leq \frac{1}{\Gamma(q)} \int_{t_{0}}^{t} \frac{d\left(f\left(s, \widehat{x}_{n}(x)\right), \widetilde{0}\right) d s}{(t-s)^{-q+1}},
\end{aligned}
$$

which lead to derive:

$$
\begin{aligned}
& d\left(x_{1}(t), x^{0}(t)\right) \leq\left.\frac{M}{\Gamma(q)} \frac{(t-s)^{q}}{-q}\right|_{t_{0}} ^{t} \leq b, \\
& d\left(\widehat{x}_{1}(t), x^{0}(t)\right) \leq\left.\frac{M}{\Gamma(q)} \frac{(t-s)^{q}}{-q}\right|_{t_{0}} ^{t} \leq b .
\end{aligned}
$$

By using induction, these present that $\left\{x_{n+1}(t)\right\}$ and $\left\{\widehat{x}_{n+1}(t)\right\}$ are uniformly bounded on $\left[t_{0}, t_{0}+\eta\right]$.

In order to show that $\phi(t)$ is continuous, please see the Appendix.

It is easy to verify that $x_{n+1}(t)$ is equicontinuous on $\left[t_{0}, t_{0}+\eta\right]$ using the lower and upper functions of $x_{n+1}(t)$, represented by $\underline{x}_{n+1}(t ; r)$ and $\bar{x}_{n+1}(t ; r)$, respectively. Therefore, for proving Theorem, we have to show that:

$\phi(t)=\limsup _{n \rightarrow \infty} d\left(x_{n+1}(t), x_{n}(t)\right) \equiv 0, \quad t \in\left[t_{0}, t_{0}+\eta\right]$.
Similarly, for the family of functions $\widehat{x}_{n+1}(t)$, it is sufficient to show that:

$\widehat{\phi}(t)=\limsup _{n \rightarrow \infty} d\left(\widehat{x}_{n+1}(t), \widehat{x}_{n}(t)\right) \equiv 0, \quad t \in\left[t_{0}, t_{0}+\eta\right]$.

Now, by using the fuzzy version of Nagumo condition (3) and the definitions of $x_{n+1}(t)$ and $\widehat{x}_{n+1}(t)$ given by (4) and (5), respectively, we obtain:

$d\left(x_{n+1}(t), x_{n}(t)\right) \leq \frac{q \Gamma(q)}{\Gamma(q)} \int_{t_{0}}^{t}(t-s)^{q} \frac{d\left(x_{n}(s), x_{n-1}(s)\right)}{\left(s-t_{0}\right)^{q}} d s$,

and

$d\left(\widehat{x}_{n+1}(t), \widehat{x}_{n}(t)\right) \leq \frac{q \Gamma(q)}{\Gamma(q)} \int_{t_{0}}^{t}(t-s)^{q} \frac{d\left(\widehat{x}_{n}(s), \widehat{x}_{n-1}(s)\right)}{\left(s-t_{0}\right)^{q}} d s$.

For a fixed $t$ in $\left[t_{0}, t_{0}+\eta\right]$, there is a sequence of integers $n_{1}<n_{2}<\ldots$ such that:

$$
\begin{aligned}
& d\left(x_{n+1}(t), x_{n}(t)\right) \longrightarrow \phi(t) \text { as } n=n_{k} \longrightarrow \infty \\
& d\left(\widehat{x}_{n+1}(t), \widehat{x}_{n}(t)\right) \longrightarrow \widehat{\phi}(t) \text { as } n=n_{k} \longrightarrow \infty .
\end{aligned}
$$

Setting

$$
\begin{aligned}
& \phi_{*}(t)=\lim _{n=n_{k} \rightarrow \infty} d\left(x_{n}(t), x_{n-1}(t)\right), \\
& \widehat{\phi}_{*}(t)=\lim _{n=n_{k} \rightarrow \infty} d\left(\widehat{x}_{n}(t), \widehat{x}_{n-1}(t)\right) .
\end{aligned}
$$

exist uniformly on $\left[t_{0}, t_{0}+\eta\right]$. Therefore,

$$
\begin{aligned}
\phi(t) & \leq q \cdot \int_{t_{0}}^{t}(t-s)^{q-1} \frac{\phi_{*}(s)}{\left(s-t_{0}\right)^{q}} d s, \\
\widehat{\phi}(t) & \leq q \cdot \int_{t_{0}}^{t}(t-s)^{q-1} \frac{\widehat{\phi}_{*}(s)}{\left(s-t_{0}\right)^{q}} d s,
\end{aligned}
$$

which lead to obtain the following results using the fact that $\phi_{*}(t) \leq \phi(t)$ and $\widehat{\phi}_{*}(t) \leq \widehat{\phi}(t)$ :

$$
\begin{aligned}
\phi(t) & \leq q \cdot \int_{t_{0}}^{t}(t-s)^{q-1} \frac{\phi(s)}{\left(s-t_{0}\right)^{q}} d s, \\
\widehat{\phi}(t) & \leq q \cdot \int_{t_{0}}^{t}(t-s)^{q-1} \frac{\widehat{\phi}(s)}{\left(s-t_{0}\right)^{q}} d s .
\end{aligned}
$$

It is easy to verify that:

$$
\begin{aligned}
& \frac{\phi(t)}{t-t_{0}} \longrightarrow 0, \quad \text { as } t \longrightarrow t_{0}^{+}, \\
& \frac{\widehat{\phi}(t)}{t-t_{0}} \longrightarrow 0, \quad \text { as } \quad t \longrightarrow t_{0}^{+},
\end{aligned}
$$

and, also, it is easy to show that:

$$
\begin{aligned}
& \frac{\phi(t)}{\left(t-t_{0}\right)^{q}} \longrightarrow 0, \quad \text { as } t \longrightarrow t_{0}^{+}, \\
& \frac{\widehat{\phi}(t)}{\left(t-t_{0}\right)^{q}} \longrightarrow 0, \quad \text { as } t \longrightarrow t_{0}^{+} .
\end{aligned}
$$

Setting $\psi(t)=\frac{\phi(t)}{\left(t-t_{0}\right)^{q}}$. Note that $\psi\left(t_{0}\right) \equiv 0$. We shall prove that $\psi(t) \equiv 0$ for all $t$ in $\left[t_{0}, t_{0}+\eta\right]$ (12) for demonstrating the convergence of $\left\{x_{n+1}(t)\right\}$ and 
$\left\{\widehat{x}_{n+1}(t)\right\}$. If it is not true, let $m=\psi\left(t_{1}\right)=$ $\max _{\left[t_{0}, t_{0}+\eta\right]} \psi(t)$ and $\widehat{m}=\widehat{\psi}\left(t_{2}\right)=\max _{\left[t_{0}, t_{0}+\eta\right]} \widehat{\psi}(t)$.

Then, we have:

$$
\begin{aligned}
m & =\psi\left(t_{1}\right) \\
& =\frac{\phi(t)}{\left(t-t_{0}\right)^{q}} \\
& \leq \frac{q}{\left(t_{1}-t_{0}\right)^{q}} \int_{t_{0}}^{t_{1}}\left(t_{1}-s\right)^{q-1} \frac{\phi(s)}{\left(s-t_{0}\right)^{q}} d s \\
& <q\left(t_{1}-t_{0}\right)^{-q} \int_{t_{0}}^{t_{1}}\left(t_{1}-s\right)^{q-1} \psi(s) d s \\
& <q m\left(t_{1}-t_{0}\right)^{-q} \frac{\left(t_{1}-t_{0}\right)^{q}}{q}=m,
\end{aligned}
$$

and

$$
\begin{aligned}
\widehat{m} & =\widehat{\psi}\left(t_{2}\right) \\
& =\frac{\widehat{\phi}(t)}{\left(t-t_{0}\right)^{q}} \leq \frac{q}{\left(t_{2}-t_{0}\right)^{q}} \int_{t_{0}}^{t_{2}}\left(t_{2}-s\right)^{q-1} \frac{\widehat{\phi}(s)}{\left(s-t_{0}\right)^{q}} d s \\
& <q\left(t_{2}-t_{0}\right)^{-q} \int_{t_{0}}^{t_{2}}\left(t_{2}-s\right)^{q-1} \widehat{\psi}(s) d s \\
& <q m\left(t_{2}-t_{0}\right)^{-q} \frac{\left(t_{2}-t_{0}\right)^{q}}{q}=\widehat{m}
\end{aligned}
$$

which are contradictions. The proof is complete.

\section{Krasnoselskii-Krein-type uniqueness for FFDEs}

Here, we extend the fuzzy Krasnoselskii-Krein-type uniqueness result for FFDE (1).

Theorem 5 Assume that the fuzzy-valued function $f$ in (1) satisfies the following Krasnoselskii-Kreintype conditions:

$$
\text { (A) } d(f(t, x), f(t, y)) \leq \frac{k r \Gamma(q) d(x, y)}{\left(t-t_{0}\right)^{q}},
$$

for $t \neq t_{0}, k r \leq q, k>1$.

$$
\text { (B) } d(f(t, x), f(t, y)) \leq C[d(x, y)]^{\alpha},
$$

for $C$ is constant, $0<\alpha<1$ and $k(1-\alpha)<1$.

Then, the following successive approximations given by

$x_{n+1}(t)=x^{0}(t)+\frac{1}{\Gamma(q)} \int_{t_{0}}^{t}(t-s)^{q-1} f\left(s, x_{n}(s)\right) d s$

in the sense of $(1, q)$-differentiability, and

$$
\widehat{x}_{n+1}(t)=x^{0}(t) \ominus \frac{-1}{\Gamma(q)} \int_{t_{0}}^{t}(t-s)^{q-1} f\left(s, \widehat{x}_{n}(s)\right) d s
$$

in the sense of $(2, q)$-differentiability, converge to two unique solutions $x(t)$ and $\widehat{x}(t)$ of (1), respectively, on $\left[t_{0}, t_{0}+\eta\right]$ where $(t, x),(t, y) \in \mathbb{E}_{0}, \eta=$ $\min =\left(a,\left(\frac{b \Gamma(1+q)}{M}\right)^{\frac{1}{q}}\right), M$ being the bound of $f$ on $\mathbb{E}_{0}$, i.e., $d(f, \widetilde{0}) \leq M$.
Proof. Let us consider $x(t), y(t)$ be any two $(1, q)$ solutions of (1). Let us consider

$$
\phi(t)=d(x(t), y(t)) .
$$

Note that $\phi\left(t_{0}\right) \equiv 0$. Also, using (14) and condition (B), we have:

$\phi(t)=d(x(t), y(t)) \leq \frac{C}{\Gamma(q)} \int_{t_{0}}^{t}(t-s)^{q-1}(\phi(s))^{\alpha} d s$.

Consider $R(t)=\frac{C}{\Gamma(q)} \int_{t_{0}}^{t}(t-s)^{q-1}(\phi(s))^{\alpha} d s$. We show that $R(t)$ is

$$
R(t)=L\left(t-t_{0}\right)^{\beta},
$$

where unknown parameters $L, \beta>0$ are to be determined such that:

$$
\left({ }^{R L} D^{q} R\right)(t)=(R(t))^{\alpha} .
$$

By using Definition 4, we get:

$$
\begin{aligned}
\left({ }^{R L} D^{q} R\right)(t) & =\frac{1}{\Gamma(1-q)} \frac{d}{d t}\left(\int_{t_{0}}^{t}(t-s)^{-q}(t-s)^{\beta} d s\right) \\
& =\frac{1}{\Gamma(1-q)} \frac{d}{d t}\left(\int_{t_{0}}^{t}(t-s)^{\beta-q} d s\right) \\
& =\frac{L}{\Gamma(1-q)}\left(t-t_{0}\right)^{\beta-q} .
\end{aligned}
$$

The last result holds while

$$
\frac{L}{\Gamma(1-q)}\left(t-t_{0}\right)^{\beta-q}=L^{\alpha}\left(t-t_{0}\right)^{\beta \alpha} .
$$

Thus, we obtain parameters $L, \beta$ as follows:

$$
L=(\Gamma(1-q))^{\frac{1}{1-\alpha}}, \quad \beta=\frac{q}{1-\alpha} .
$$

Hence, we get:

$$
\phi(t) \leq R(t)
$$

and also,

$$
\frac{\phi(t)}{\left(t-t_{0}\right)^{k q}} \leq L\left(t-t_{0}\right)^{\beta-k q} .
$$

Therefore, $\psi(t)=\frac{\phi(t)}{\left(t-t_{0}\right)^{k q}}$ is defined and also $\psi\left(t_{0}\right)=0$.

By using condition $(A)$, we obtain:

$$
\begin{aligned}
\phi(t) & =d(x(t), y(t)) \\
& \leq \frac{1}{\Gamma(q)} \int_{t_{0}}^{t}(t-s)^{q-1} d(f(s, x(s)), f(s, y(s))) d s \\
& \leq k r \int_{t_{0}}^{t}(t-s)^{q-1} \frac{\phi(s)}{\left(s-t_{0}\right)^{q}} d s \\
& =k r \int_{t_{0}}^{t}(t-s)^{q-1}\left(s-t_{0}\right)^{k q-q} \psi(s) d s .
\end{aligned}
$$

Now, we should prove that $\phi(t) \equiv 0$ which is completely similar to crisp case [12], hence it is omitted. 


\section{Comparison results}

In this section, we compare the obtained results with together for obtaining useful relations.

Theorem 6 Let us consider

$H_{1}=\min _{\left[t_{0}, t_{0}+\eta\right]}\left\{C(d(x(t), y(t)))^{\alpha}, \frac{k r \cdot \Gamma(q) \cdot d(x(t), y(t))}{\left(t-t_{0}\right)^{q}}\right\}$,
$H_{2}=\max _{\left[t_{0}, t_{0}+\eta\right]}\left\{C(d(x(t), y(t)))^{\alpha}, \frac{k r \cdot \Gamma(q) \cdot d(x(t), y(t))}{\left(t-t_{0}\right)^{q}}\right\}$,

then, under assumptions of Theorem 4 and Theorem 5, we get:

(1) if $\mathrm{H}_{2}<\frac{q \cdot \Gamma(q) \cdot d(x(t), y(t))}{\left(t-t_{0}\right)^{q}}$ and Theorem 5 holds, then Theorem 4 holds.

(2) if $H_{1}>\frac{q \cdot \Gamma(q) \cdot d(x(t), y(t))}{\left(t-t_{0}\right)^{q}}$ and Theorem 4 holds, then Theorem 5 holds.

Proof. By using fuzzy version of Nagumo condition and Krasnoselskii-Krein conditions, the proofs are obvious.

Theorem 7 Under assumptions of Theorem 4 and Theorem 5, if there exists a constant $C$ such that:

$C(d(x(t), y(t)))^{\alpha} \leq \frac{k r \cdot \Gamma(q) \cdot d(x(t), y(t))}{\left(t-t_{0}\right)^{q}}, \quad t \in\left[t_{0}, t_{0}+\eta\right]$.

Then, if Theorem 5 holds, Theorem 4 holds.

Proof. By using the fact that $K r \leq q$, the proof is straightforward.

\section{Conclusion}

In this paper, we considered two new uniqueness results for fuzzy fractional differential equations (FFDEs) involving Riemann-Liouville generalized H-differentiability with fuzzy version of Nagumoand Krasnoselskii-Krein conditions. To this purpose, we obtained the equivalent integral forms of original FFDE and then these are used to investigate convergence of corresponding successive approximations. Finally, some useful comparison results obtained to show the conditions that when Theorem 4 has some benefit than Theorem 5 and vice versa.

\section{References}

[1] S. Abbasbandy, A. Shirzadi, Homotopy analysis method for multiple solutions of the fractional Sturm-Liouville problems, Numer. Algor. 54 (2010) 521-532.

[2] R.P. Agarwal, V. Lakshmikantham, Uniqueness and Nonuniqueness Criteria for Ordinary Differential Equations, World Scientific, Singapore, 1993.
[3] R.P. Agarwal, V. Lakshmikantham, J.J. Nieto, On the concept of solution for fractional differential equations with uncertainty, Nonlinear Anal. 72 (2010) 2859-2862.

[4] A. Arara, M. Benchohra, N. Hamidi, J.J. Nieto, Fractional order differential equations on an unbounded domain, Nonlinear Anal. 72 (2010) 580-586.

[5] B. Bede, S.G. Gal, Generalizations of the differentiability of fuzzy-number-valued functions with applications to fuzzy differential equations, Fuzzy Sets and Systems 151 (2005) 581599.

[6] K. Diethelm, N.J. Ford, Analysis of fractional differential equations, J. Math. Anal. Appl. 265 (2002) 229-248.

[7] K. Diethelm, N.J. Ford, Multi-order fractional differential equations and their numerical solution, AMC 154 (2004) 621-640.

[8] M. Friedman, M. Ma, A. Kandel, Numerical solution of fuzzy differential and integral equations, Fuzzy Sets and System 106 (1999) 35-48.

[9] A.A. Kilbas, H.M. Srivastava, J.J. Trujillo, Theory and Applications of Fractional Differential Equations, Elsevier Science B.V, Amesterdam, 2006.

[10] O. Kooi, The method of successive approximations and a uniqueness theorem for Krasnoselskii-Krein in the theory of differential equations, Nederi. Akad. Wetensch, Proc. Ser. A61; Indag. Math. 20 (1958) 322-327.

[11] V. Lakshmikantham, S. Leela, Nagumo-type uniqueness result for fractional differential equations, J. Nonlinear Anal. 71 (7-8) (2009) 2886-2889.

[12] V. Lakshmikantham, S. Leela, KrasnoselskiiKrein type uniqueness result for fractional differential equations, J. Nonlinear Anal. TMA71 (7-8) (2009) 3421-3424.

[13] V. Lakshmikantham, S. Leela, J. Vasundhara Devi, Theory of Fractional Dynamic Systems, Cambridge Scientific Pub, Cambridge, UK, 2009.

[14] V. Lakshmikantham, R.N. Mohapatra, Theory of Fuzzy Differential Equations and Applications, Taylor \& Francis, London, 2003.

[15] V. Lakshmikantham, A.S. Vatsala, Basic theory of fractional differential equations, Nonlinear Anal. 69 (2008) 2677-2682.

[16] M. Ma, M. Friedman, A. Kandel, Numerical solution of fuzzy differential equations, Fuzzy Sets and Systems 105 (1999) 133-138.

[17] I. Podlubny, Fractional Differential Equation, Academic Press, San Diego, 1999.

[18] M.L. Puri, D. Ralescu, Fuzzy random variables, J. Math. Anal. Appl. 114 (1986) 409-422.

[19] H.C. Wu, The improper fuzzy Riemann integral and its numerical integration, Information Sciences 111 (1999) 109-137.

[20] J. Xu, Z. Liao, Z. Hu, A class of linear dif- 
ferential dynamical systems with fuzzy initial condition, Fuzzy Sets and Systems 158 (2007) 2339-2358.

[21] H.J. Zimmermann, Fuzzy set theory and its applications, Kluwer Academic Publishers, Dordrecht, 1991.

\section{Appendix}

For $t_{0} \leq t_{1} \leq t_{2}$, we have:

$$
\begin{aligned}
d\left(x_{n}\left(t_{1}\right), x_{n-1}\left(t_{1}\right)\right) & -d\left(x_{n}\left(t_{2}\right), x_{n-1}\left(t_{2}\right)\right) \\
& \leq\left|d\left(x_{n}\left(t_{1}\right), x_{n-1}\left(t_{1}\right)\right)-d\left(x_{n}\left(t_{2}\right), x_{n-1}\left(t_{2}\right)\right)\right| \\
& \leq \frac{1}{\Gamma(q)}\left[\left|\int_{t_{0}}^{t_{1}}\left(t_{1}-s\right)^{q-1} D(s) d s-\int_{t_{0}}^{t_{2}}\left(t_{2}-s\right)^{q-1} D(s) d s\right|\right] \\
& =\frac{1}{\Gamma(q)}\left[\left|\int_{t_{0}}^{t_{1}}\left\{\left(t_{1}-s\right)^{q-1}-\left(t_{2}-s\right)^{q-1}\right\} D(s) d s-\int_{t_{1}}^{t_{2}}\left(t_{2}-s\right)^{q-1} D(s) d s\right|\right] \\
& \leq \frac{2 M}{\Gamma(q)}\left[\left|\int_{t_{0}}^{t_{1}}\left\{\left(t_{1}-s\right)^{q-1}-\left(t_{2}-s\right)^{q-1}\right\} d s+\int_{t_{1}}^{t_{2}}\left(t_{2}-s\right)^{q-1} d s\right|\right] \\
& =\frac{2 M}{q \Gamma(q)}\left[\left|\left(t_{1}-t_{0}\right)^{q}-\left(t_{2}-t_{0}\right)^{q}+2\left(t_{2}-t_{1}\right)^{q}\right|\right] \\
& \leq \frac{4 M}{q \Gamma(q)}\left(t_{2}-t_{1}\right)^{q}=\frac{4 M}{\Gamma(q+1)}\left(t_{2}-t_{1}\right)^{q},
\end{aligned}
$$

where $D(s)=d\left(f\left(s, x_{n-1}(s)\right), f\left(s, x_{n-2}(s)\right)\right) \leq 2 M$ on $\mathbb{E}_{0}$. Note that the last obtained result can be made less than $\epsilon>0$ provided that $\left|t_{2}-t_{1}\right|<\delta=$ $\left(\frac{\epsilon \Gamma(1+q)}{4 M}\right)^{\frac{1}{q}}$. This leads to obtain:

$$
\left|\phi\left(t_{1}\right)-\phi\left(t_{2}\right)\right| \leq \epsilon,
$$

which proves the continuity of $\phi(t)$. Similar discussion holds for continuity of $\widehat{\phi}(t)$, hence it is omitted. Please notice that the idea of using mentioned uniqueness theorems was discussed in the deterministic case for fractional differential equations in $[10,11,12]$. 